\title{
IV. Innere und äußere Rahmenbedingungen der „Vergangenheitsbewältigung“
}

Über den zentralen, auch wirkungsgeschichtlichen Unterschied zwischen Hirohito und Hitler hinaus differierten weitere Ausgangsbedingungen des japanischen Umgangs mit der Vergangenheit fundamental von den deutschen. Am Ende des Krieges standen die alliierten Soldaten, nachdem sie die Wehrmacht aus den besetzten Gebieten zurückgeworfen und monatelang Quadratmeter um Quadratmeter deutschen Bodens erobert hatten, gemeinsam mitten im Reich. Die Niederlage des NS-Staates war total, die Kapitulation am 8. Mai 1945 bedingungslos, jede staatliche Autorität zusammengebrochen, ein Viertel des angestammten deutschen Siedlungsgebiets östlich von Oder und Neiße verloren. Zum Zeitpunkt der Verkündung der Kapitulationsbereitschaft Japans am 15. August 1945 befand sich dagegen kein einziger fremder Soldat auf dessen Mutterboden; Wochen verstrichen, bis es dahin kam, und daß während der besatzungslosen Tage politische Absprachen getroffen wurden, die der Haltung des Landes gegenüber der Siegermacht ein gewisses $\mathrm{Maß}$ an Kohärenz sicherten ${ }^{1}$, ist unübersehbar ${ }^{2}$. Obwohl die Niederlage für die meisten Japaner völlig unerwartet gekommen war und der 15. August 1945 von der auf Dorfplätzen und Schulhöfen, Fabriken und Kasernen versammelten Bevölkerung auch insoweit als historische Zäsur erlebt wurde, als alle zum ersten Mal die Stimme des Tennô Hirohito mit eigenen Ohren vernahmen ${ }^{3}$, konnte von einem "Nullpunkt" und von völligem Neubeginn tatsächlich nicht die Rede sein. Japan wurde zwar erstmals seit dem Altertum wieder von einer fremden Macht beherrscht, doch der Staat selbst blieb erhalten - und auch eine funktionsfähige Regierung als Juniorpartner der Besatzungsmacht. In der gewundenen Proklamation des Kaisers über die Beendigung des Krieges findet sich das Wort „Kapitulation" denn auch an keiner Stelle. Hinzu kommt, daß Japan nicht wie Deutschland in vier Besatzungszonen aufgeteilt wurde, sondern allein die Amerikaner das Reich des Tennô okkupierten. Die von den Sowjets angestrebte Mitbesetzung der Nordinsel Hokkaidô scheiterte am Widerstand der USA, die bereits erste Erfahrungen mit der schwierigen Vier-Mächte-

1 Vgl. Ball, Japan, S. 47.

2 Zu den Schwierigkeiten, konkrete Beweise dafür zu finden, vgl. Gasteyger, Das außenpolitische Erbe, S. 173.

3 Buchholz, Demokratisierung der Erinnerung, S. 48. 
Verwaltung in Deutschland gesammelt hatten. Vom beträchtlichen Kolonialbesitz abgesehen gingen nur die winzigen Südkurilen-Inseln an Rußland verloren ${ }^{4}$, die Ryukyu-Inseln im Süden (mit Okinawa) kamen unter amerikanische Militärverwaltung.

Länger als auf Japan, über ein Jahrzehnt hinweg, behielten die vier Siegermächte auf das besetzte Deutschland - noch dazu weiterreichenden Durchgriff. Jenen Grad an eingeschränkter staatlicher Eigenständigkeit, der für Japan zwischen 1945 und 1952 charakteristisch gewesen war, erreichte Westdeutschland erst mit der Gründung der Bundesrepublik und der Einführung des Besatzungsstatuts 1949. Und diese Phase reduzierter Souveränität hielt - wenn auch mit zunehmenden Modifizierungen ${ }^{5}$ - bis 1955 an, während sie Japan 1952 mit dem Abschluß des Friedensvertrages zumindest ein Stück weiter hinter sich ließ6. Selbst nach dem 5. Mai 1955 blieb die Bundesregierung obendrein zumindest in ihrer deutschlandpolitischen Handlungsfreiheit dauerhaft eingeschränkt, weil die vier Siegermächte bis zu einem unabsehbaren Friedensvertrag Mitspracherechte in bezug auf Deutschland als Ganzes behielten.

Der für Japan nach dem Zweiten Weltkrieg eingerichtete Alliierte Rat mit Sitz in Tokio, der aus Vertretern der USA, der Sowjetunion, Chinas und Englands bestand, hatte dagegen ebenso wie die aus elf Ländern beschickte Fernost-Kommission nur beratende Funktion. Als beide Gremien im Frühjahr 1946 ihre Arbeit aufnahmen, waren die grundlegenden Verfügungen zur Demokratisierung Japans vom General Headquarter des SCAP bereits ausgearbeitet. Und MacArthur dachte ohnehin nicht daran, sich von den anderen Siegermächten in einem Aktionsradius beschneiden zu lassen, der schon deshalb enorm war, weil sich das ihm von Präsident Truman am 29. August 1945 übermittelte Konzept „U.S. Initial Post-Surrender Policy toward Japan“ (mit den Prinzipien der „Demokratisierung“ und „Entmilitarisierung ") als ausgesprochen dehnbar erwies ${ }^{7}$ - zumal in der Zeit, als in Washington die sicherheitspolitisch denkende China-Lobby im Verteidigungsministerium mit der Japan-Lobby im Außenministerium stritt.

Die Abberufung des konservativen Japan-Verehrers und früheren Botschafters in Tokio, Joseph Grew, von seinem Amt als Unterstaatssekretär für Asien im Außenministerium am 11. August 1945 verschob in der USAdministration eine Zeit lang die Gewichte zu Gunsten der China-Lobby.

4 Allerdings auch das 1905 eroberte Südsachalin.

5 Den „Hürdenlauf zur westdeutschen Souveränität“ in den Jahren 1952-1955 analysiert instruktiv Vogt, Wächter der Bonner Republik, S. $215 \mathrm{ff}$.

6 Auch das Vertragswerk von San Francisco begründete indes, wie John W. Dower herausgestellt hat, keine wirkliche Souveränität für Japan, sondern verurteilte das Land militärisch und diplomatisch zur Zweitklassigkeit. Dower, Yoshida in the scales of history, S. 208-241. Erst 1972 wurde zudem die - 1969 vereinbarte - Rückkehr Okinawas unter japanische Souveränität Wirklichkeit.

7 Vgl. Hata, Japan, S. 197. 
Unterstützt von Grews liberalem Nachfolger Dean Acheson setzte man auf ein unter der Kuomintang geeintes Reich der Mitte als stabilisierende antibolschewistische Macht Nachkriegsasiens. Den Japanern dagegen war nach diesem Szenario - vielleicht auch durch die Abschaffung des Tennô-Systems - der Militarismus möglichst gründlich auszutreiben. Die selbst am General Headquarter des SCAP nicht ganz spurlos vorübergehende Verhärtung der amerikanischen Japanpolitik sollte indes nicht von langer Dauer sein. Schon im Frühjahr 1946 war das Scheitern der Vermittlungsmission des nach China gesandten US-Präsidialbotschafters General George C. Marshall offensichtlich, als erbitterte Kämpfe zwischen Kuomintang und Kommunisten ausbrachen ${ }^{8}$. Die Ausweitung des chinesischen Bürgerkriegs und schließlich der Sieg Mao Zedongs über Jiang Kaishek (1949) zwangen die USA zu einer grundlegenden Neubewertung - und das hieß: einer Aufwertung - der künftigen außenpolitischen Rolle Japans, was sich mehr und mehr auch auf den Umgang der USA mit der ultranationalistischen Vergangenheit auswirkte.

Der Inselstaat, der sich bis zum Ende des Krieges der Sowjetunion gegenüber neutral verhalten, ja zum Schluß sogar auf deren diplomatische Vermittlungsdienste gesetzt hatte, schien aufgrund der jüngsten Geschichte weniger gegen die kommunistische Gefahr immunisiert als der freie Teil Deutschlands, wo nicht nur die antibolschewistische Propaganda der Nationalsozialisten, sondern auch die Erfahrung mit den Exzessen der Roten Armee stärker nachwirkte - von dem anhaltenden kommunistischen Anschauungsunterricht in der geknebelten SBZ einmal ganz abgesehen. Die wachsende Notwendigkeit, Japan zu einem Bollwerk gegen den Kommunismus auszubauen, der sich an der pazifischen Gegenküste der USA - mit dem sowjetischen und chinesischen Koloß im Rücken - bald nach Korea und Vietnam auszubreiten anschickte, bedeutete das Überdenken jener politischen Richtlinien, die MacArthur Ende August 1945 auf dem Flug nach Japan Generalmajor Courtney Whitney diktiert hatte.

Diese erste Konzeption hatte von der Zerstörung des Militärpotentials, der Befreiung der Bauern und Entlassung der politischen Gefangenen über die Schaffung freier Gewerkschaften, die Beseitigung polizeilicher Unterdrückung und die Förderung einer freien Wirtschaft bis hin zur Entwicklung einer freien und verantwortungsbewußten Presse, Liberalisierung des Erziehungswesens, Einführung des Frauenwahlrechts und politischer Dezentralisierung gereicht ${ }^{9}$. Nur drei Jahre später, im entscheidenden Wendejahr 1948, billigte Präsident Truman (im Oktober) drastisch veränderte, vom Nationalen Sicherheitsrat beschlossene Maximen für die amerikanische Japanpolitik. In der sog. Direktive NSC 13/2 kam die Auffassung der dezi-

9 Whitney, MacArthur, S. 213. 
diert antikommunistischen Containment-Politiker zum Ausdruck, wonach China-Lobby und "rote“ New Dealer - auch im General Headquarter Japan mit ihren Reformen massiv geschwächt, eine Arbeitslosigkeit von 20 Prozent und eine Inflationsrate von 50 Prozent mit zu verantworten und damit die Gefahr einer Revolution heraufbeschworen hätten. Die Besatzung müsse folglich so lange aufrechterhalten werden, bis das Land wirtschaftlich wieder auf eigenen Füßen stehe. Dem Chef-Planer im US-Außenministerium, George F. Kennan, war es im Vier-Augen-Gespräch mit MacArthur in Tokio gelungen, den General von der Notwendigkeit eines Kurswechsels zu überzeugen, nachdem dieser sich auch selbst schon veranlaßt gesehen hatte, einen kommunistisch inspirierten Generalstreik zu verbieten ${ }^{10}$. Ganz im Mittelpunkt der US-Besatzungspolitik stand künftig die ökonomische Stabilisierung Japans: Das General Headquarter sollte die Reformgesetzgebung so schnell wie möglich den Japanern überlassen, die Polizei umorganisiert und verstärkt und die laufende politische Säuberung abgemildert werden, da infolge dieser Maßnahmen vor allem im Erziehungswesen die Kommunisten zu stark geworden seien (im Kennan-Bericht war ursprünglich sogar von einer Einstellung der Säuberungen die Rede) ${ }^{11}$.

Der neue Kurs der amerikanischen Japan-Politik wurde auch dadurch begünstigt, daß Nippon für die USA in gewisser Weise fast noch höhere strategische Bedeutung gewann als Westdeutschland. Jedenfalls gab es in Europa mit England und Frankreich auch noch andere große Bündnispartner, die indes schwere Opfer im Kampf gegen den Nationalsozialismus gebracht hatten und nun ihrerseits ein scharfes Auge auf den deutschen Umgang mit der braunen Erblast warfen, man denke nur daran, wie 1950 ein britischer Außenminister im Unterhaus über Parallelen zwischen Nationalsozialismus und deutschem Wesen philosophierte oder die französische $\mathrm{Na}$ tionalversammlung über die Pflege der Gräber von Dachauer KZ-Opfern beriet $^{12}$. In Ostasien dagegen kam spätestens nach dem kommunistischen Sieg in China eigentlich nur noch Japan als Hauptbastion der freien Welt in Frage. Und nicht nur das Interesse der USA an japanischer „Vergangenheitsbewältigung " war aufgrund dieser Konstellation gedämpft; hinzu kam, daß sich nach 1949 sowohl Rot- als auch Nationalchina mit Forderungen an Japan zurückhielten, weil für beide rivalisierende Regierungen das Bemühen um Handelsbeziehungen und politische Anerkennung durch Tokio im Vordergrund stand. Für das kleine Volk der Koreaner, das fürchterlich unter dem japanischen Imperialismus gelitten hatte, galt ähnliches. Andere im Krieg schwer geschädigte Länder des ostasiatischen Raumes waren ebenfalls lange sehr stark mit sich, d.h. vor allem mit dem Prozeß der Dekolonisie-

10 Scharlau, Der General und der Kaiser, S. 108.

11 Hata, Japan, S. $209 \mathrm{ff}$.

12 Kittel, Dic Legende, S. 374. 
rung und der Entwicklung eigener, neuer Identitäten beschäftigt ${ }^{13}$. So brachten die Opfer der japanischen Aggression insgesamt offensichtlich ungleich weniger „bewältigungspolitisches“ Gewicht auf die Waage als Engländer, Franzosen, Israelis oder jüdische Organisationen in den USA.

Zudem waren freundschaftliche Beziehungen zwischen der Bundesrepublik und den westlichen Nachbarn, die nur auf der Basis einer glaubwürdigen Bewältigung der NS-Vergangenheit möglich werden konnten, auch im Hinblick auf den nur gemeinsam zu bewerkstelligenden wirtschaftlichen Wiederaufbau der vom Krieg schwer gezeichneten Länder unerläßlich. Die ökonomische Konsolidierung Japans dagegen erfolgte nicht durch den $\mathrm{Zu}-$ sammenschluß mit asiatischen Nachbarländern, nicht qua Montanunion und supranationaler Wirtschaftsgemeinschaft, sondern vor allem in bilateraler Verbindung mit den USA.

Nicht nur größere Rücksicht auf stärkere westeuropäische Verbündete richtete den amerikanischen Fokus mit mehr Schärfe auf die deutsche „Vergangenheitsbewältigung" als auf die japanische, sondern auch eine in mehrfacher Hinsicht viel engere Beziehung zur größten Opfergruppe der NSDiktatur. Zum einen spielte der 1948 gegründete Staat Israel, dessen Entstehung unmittelbar zur Wirkungsgeschichte des Holocaust gehört, im geostrategisch wichtigen Nahen Osten schon bald eine herausgehobene Rolle als Verbündeter der freien Welt. Aber auch vorher war „das jüdische Element als bedeutsamer innenpolitischer Faktor" 14 in den USA - nach Curt Gasteyger - gerade bei der Formulierung und Durchführung der amerikanischen Deutschlandpolitik bereits zu berücksichtigen. Historisch unangemessen wäre es allerdings, diesen Faktor absolut zu setzen, weil seine Wirkungen auf die US-Außenpolitik ebenso wie auf die bundesdeutsche „Bewältigungspolitik“ sich nur in einem höchst komplexen Bedingungsgeflecht entfalten konnten.

Zunächst ist zu sehen, daß der Holocaust in den ersten zwei Jahrzehnten nach 1945 in der us-amerikanischen Erinnerungskultur alles andere als eine zentrale Rolle spielte. Schon 1947 kritisierte der Vertreter des American Jewish Committee ${ }^{15}$ in Washington die politische Klasse des Landes, weil sie sich für Deutschland „nur als Bollwerk gegen den Bolschewismus“ interessiere und der nationalsozialistischen Vergangenheit „völlig gleichgültig gegenüber" stehe ${ }^{16}$. Als symptomatisch kann gelten, daß ein 1947 in New York geplantes Holocaust-Denkmal im Manhattaner Riverside Park nach einer über zehn Jahre dauernden Diskussion mit Rücksicht auf „die anderen

13 Vgl. Acharya, The Quest for Identity; Frey, Drei Wege zur Unabhängigkeit.

14 Gasteyger, Das außenpolitische Erbe, S. 170.

15 Der Verband war 1906 „von deutschstämmigen Juden der oberen amerikanischen Mittelschicht" gegründet worden und blieb für viele Jahre „die Gruppe mit dem größten Einfluß auf die US-Regierung“. Shafir, Die Rolle der Amerikanisch-Jüdischen Organisationen, S. 55.

16 Novick, Nach dem Holocaust, S. 124. 
gesellschaftlichen Randgruppen "17 niemals gebaut wurde. Den Protesten der - noch dazu ausgesprochen heterogenen - amerikanisch-jüdischen Organisationen gegen Defizite bundesdeutscher „Vergangenheitsbewältigung“ haftete in den ersten Dekaden nach 1945 laut Peter Novick etwas „Rituelles und Pflichtmäßiges" 18 an, waren sie doch vor allem darum bemüht, sich auf den Leitwert eines optimistischen amerikanischen Patriotismus hin auszurichten und sich ängstlich einer Gesellschaft anzupassen, in der die noch immer verbreitete Assoziation, Jude sei gleich Kommunist, im Klima des Kalten Krieges besondere Gefahren barg ${ }^{19}$. Wegen der verdächtigen Dauerthematisierung der Shoah durch die extreme jüdische Linke habe es bei den Gemäßigten geradezu als tabu gegolten, in mit Deutschland zusammenhängenden Fragen den Holocaust zu erwähnen, „außer hinter verschlossenen Türen oder im privaten Kreise " 20.

Die kaum zu übersehenden Verdrängungstendenzen dürfen aber nicht gar zu einseitig interpretiert werden. Gerade das auch von Novick erwähnte Beispiel der Debatte um eine westdeutsche Industriemesse in New York 194921 ist nämlich nicht nur ein Beleg für das Dilemma der jüdischen Organisationen, einen Kompromiß zwischen ihren Bedenken gegen eine allzu rasche Rehabilitierung Deutschlands und den sogenannten "gesamtamerikanischen“ Wirtschafts- und Sicherheitsinteressen zu finden, sondern auch für die dann doch nicht völlig ausbleibenden Wirkungen, die aus der besonderen deutschlandpolitischen Sensibilität dieser Gruppe der amerikanischen Bevölkerung resultierten. Die für den April 1949 von der US-Militärregierung anberaumte Industriemesse zur Förderung von deutschen Exporten in die USA wurde von den jüdischen Organisationen in seltener Einmütigkeit als Provokation angesehen; nicht nur weil sich im April der Aufstand im Warschauer Ghetto jährte, sondern vor allem auch wegen des Besuchs von Industriellen, die man für mit schuldig an der Machtergreifung Hitlers hielt. Auf das vorsichtig vorgetragene Anliegen des American Jewish Committee, die Messe unbefristet aufzuschieben, reagierte General Clay zwar sehr unwirsch und verwies auf sein Einvernehmen mit „höchsten Beamten in Washington "22, versprach aber doch, die Biographien der etwa 240 Wirtschaftsführer sichten zu lassen, die nach New York fahren sollten. Die mit stiller Amtshilfe des US-Justizministeriums vorgenommene Überprüfung führte schließlich dazu, daß fast der Hälfte der Industriellen die Einladung zur Messe wegen ihrer Vergangenheit im Dritten Reich wieder entzogen wurde ${ }^{23}$.

17 So die Begründung der Beauftragten der New Yorker Kulturbehörde, zit. nach Tempel, Legenden von der Allmacht, S. 162.

18 Novick, Nach dem Holocaust, S. 124.

19 Ebd., S. $126 \mathrm{ff}$.

20 Ebd., S. 131.

21 Ebd., S. $132 \mathrm{f}$.

22 Tempel, Legenden von der Allmacht, S. 19.

23 Ebd., S. 19, 22. 
In Westdeutschland wurden die Wirkungen derartiger jüdischer Protestaktionen offensichtlich intensiver wahrgenommen als die beträchtlichen Schwierigkeiten, unter denen sie im gesellschaftlichen Klima der USA während des Kalten Krieges zustande kamen. Kaum einer dürfte damals reflektiert haben, was in vergleichender Analyse der „Vergangenheitsbewältigung" so offensichtlich scheint: Daß in Amerika nicht nur mehr einflußreiche Bürger jüdischer als chinesischer Herkunft lebten, sondern auch ungleich mehr deutsch- als japanischstämmige. Die ethnische Identität der ohnehin äußerst integrationsorientierten Deutsch-Amerikaner hatte sich zwar bereits unter den antigermanischen Schlägen infolge des Ersten Weltkriegs ${ }^{24}$ so weit aufgelöst, daß sie im Zweiten Weltkrieg nicht einmal mehr zu jenen Bevölkerungsgruppen rechneten, die in puncto staatlicher Loyalität besondere Probleme aufwarfen. Dennoch aber war das deutsch-amerikanische Verhältnis traditionell sehr viel enger als das japanisch-amerikanische. Noch dazu unterlagen die ca. 300000 Amerikaner japanischer Herkunft ${ }^{25}$ schon vor dem Zweiten Weltkrieg vielfältigen Diskriminierungen, die sich nach Pearl Harbor zu ihrer Zwangsevakuierung von der Westküste und folgender Internierung steigerten, weil an ihrer Loyalität gegenüber den USA Zweifel bestanden ${ }^{26}$. Die mindestens sechs Millionen Deutsch-Amerikaner ${ }^{27}$ bildeten dagegen zumindest ein stilles Gegengewicht gegen eine allzu drakonische US-Besatzungspolitik in Mitteleuropa. Das spiegelte sich auch in der in den USA verbreiteten Hoffnung wider, durch geeignete „chirurgische Feinarbeit" die kulturellen Schätze Deutschlands wiederbeleben zu können. Nach einer Umfrage vom Dezember 1945 meinten immerhin bereits $36 \%$ der Amerikaner, die Deutschen hätten ihre Lektion gelernt, während die Japaner nur von $20 \%$ für lernfähig gehalten wurden 28 .

Trotz all dieser Faktoren, die den potentiellen deutschlandpolitischen Einfluß der jüdischen Amerikaner begrenzten, bestimmten in der Bundesrepublik noch lange alte Stereotypen die Wahrnehmung. Selbst Konrad Adenauer, „wahrhaftig nicht vom Bazillus des Antisemitismus befallen“29,

24 Vor allem die American Protective League erzeugte seit 1917 ein Klima der Verdächtigungen, das bis zur Verbannung deutscher Musik, zur Entdeutschung von Personen- oder Städtenamen und zur öffentlichen Verbrennung deutscher Bücher führte. Obendrein hatten bis zum Sommer 1918 etwa die Hälfte der Staaten den deutschsprachigen Schulunterricht eingeschränkt oder abgeschafft. Harvard Encyclopedia, S. 423. Vgl. auch Rippley, The GermanAmericans.

25 Die Hälfte davon lebte auf Hawaii.

26 Harvard Encyclopedia, S. $565 \mathrm{ff}$. Zur Geschichte der Internierung Weglyn, Years of Infamy. Zur Entwicklung der japanischen Bevölkerungsgruppe in den USA allgemein Daniels, The Politics of Prejudice, und Hosokawa, Nisei: The Quiet Americans.

27 Zur Frage der Zahlen, die etwa in der zweiten Generation nur in Amerika Geborene „with German-born fathers" umfassen, siehe Harvard Encyclopedia, S. 406.

28 Hentschke, Demokratisierung als Ziel, S. 54.

29 Wolffsohn, Ewige Schuld?, S. 108. 
war fest davon überzeugt: „Det Judentum is eine jroße Macht“30. Bei Bedarf belehrte der Bundeskanzler auch sein Kabinett über „die große wirtschaftliche Macht des Judentums in der Welt"31. Erst recht durch die Presse der Bundesrepublik geisterte „eine milde Form der alten Legende der Weisen von Zion" 32 , wie sie von den Nationalsozialisten im Rahmen ihrer jüdischen Weltverschwörungstheorie lange genug kultiviert worden war. Auch wenn dies ein Klischee darstellte, so war es doch wirkungsmächtig.

Bei allen nötigen Differenzierungen unterliegt es jedenfalls keinem Zweifel, daß der Einfluß der 200000 Amerikaner deutsch-jüdischer Herkunft, die in den 1930er Jahren vor dem NS-Regime in die USA geflüchtet, dem Holocaust selbst also nur knapp entronnen waren, zumindest ungleich höher zu veranschlagen war als das Japan-kritische Potential der chinesischen oder koreanischen Einwanderer. Die 150000 jüdischen Einwanderer, die bereits Mitte des 19. Jahrhunderts aus deutschsprachigen Ländern gekommen waren, hatten obendrein die meisten bedeutenden Institutionen des amerikanisch-jüdischen Lebens gegründet ${ }^{33}$. Während der „middle-class character" 34 des oft zum Besitz- und Bildungsbürgertum gehörenden, insgesamt viereinhalb Millionen Menschen umfassenden amerikanischen Judentums offensichtlich war, handelte es sich bei den Chinesen um eine nicht nach Millionen zählende, sondern am Ende des Zweiten Weltkriegs gerade einmal 100000 Personen umfassende, vielfach zur Unterschicht rechnende und lange rassisch diskriminierte Minderheit. Die nicht einmal 10000 Koreaner im Land fielen natürlich noch weniger ins Gewicht.

Nachdem im Goldrausch Mitte des 19. Jahrhunderts die ersten Chinesen nach Kalifornien gekommen waren und dort für niedrigste Löhne körperliche Arbeit verrichtet hatten, wurden sie 1882 - nach Vorwürfen wegen Lohndumpings - Gegenstand des Exclusion Acts, der ihre Migration für 10 Jahre zunächst ganz stoppte ${ }^{35}$. Die dann folgende restriktive Einwanderungspolitik dauerte bis in die 1960er Jahre, auch wenn Präsident Roosevelt im Zweiten Weltkrieg das Chinese Exclusion Law als historischen Fehler bezeichnete und betonte, die Chinesen seien wegen ihres Beitrags zur alliierten Kriegführung allen anderen Asiaten vorzuziehen. Selbst in dieser Konstellation konnte sich der Kongreß aber nur zu einer jährlichen Immigrationsquote von 105 Personen durchringen, war doch die Furcht verbreitet, chinesische Einwanderer würden die amerikanische Zivilisation in ähn-

30 Tempel, Legenden von der Allmacht, S. 3 .

31 Wolffsohn, Ewige Schuld?, S. 108.

32 Tempel, Legenden von der Allmacht, S. 3.

33 Sie hatten auch etwa nach dem Urteil von Arthur Hertzberg „einen bedeutsamen Einfluß auf die amerikanische Kultur und Wissenschaft". Hertzberg, Shalom, America!, S. 10.

34 Harvard Encyclopedia, S. 589.

$35 \mathrm{Vgl}$. Chan, Entry Denied. 
licher Weise beschädigen, wie das „billige asiatische Volk“ einst den Untergang Roms herbeigeführt habe ${ }^{36}$.

Vor diesem Hintergrund wird nachvollziehbar, weshalb in bezug auf die japanischen Verbrechen während des Weltkriegs langfristig in den USA keine Entwicklung Platz greifen konnte, wie sie in den 1970er Jahren - unter grundlegend veränderten innen- und außenpolitischen Rahmenbedingungen auch infolge der Nahostkriege 1967 und 1973 - als „Amerikanisierung des Holocaust" 37 einsetzte. Chinesische Intellektuelle wie die Eltern von Iris Chang, die den Zweiten Weltkrieg in ihrer Heimat miterlebt hatten, schließlich in die USA geflohen waren und es nach einem Harvard-Studium zum Professor brachten ${ }^{38}$, gab es einfach zu wenige ${ }^{39}$, um die öffentliche Aufmerksamkeit stärker auf die von Chang untersuchten japanischen Massaker in Nanking und andernorts zu richten. Auch die Funktion Rotchinas innerhalb der außenpolitischen Strategie der Vereinigten Staaten blieb trotz der Entspannungspolitik zwischen Washington und Peking in den 1970er Jahren - offensichtlich eine grundlegend andere als die Israels. Ein besonderes Interesse an einer "Amerikanisierung von Nanking“ konnte daraus jedenfalls nicht erwachsen.

Versucht man, den Faktor des erinnerungskulturellen Einflusses jüdischer und chinesisch-stämmiger Amerikaner in eine Hierarchie von Kräften einzuordnen, die den unterschiedlichen „Außendruck“ im Prozeß der japanischen und deutschen „Vergangenheitsbewältigung" erzeugt haben, so wird ihm bei aller Bedeutung doch keineswegs die Schlüsselrolle zuzumessen sein. Er verstärkte in beiden Fällen nur noch eine Entwicklung, die vor allen Dingen in der unterschiedlichen Wirkung der Kräfte des Kalten Krieges auf Japan und Westdeutschland begründet lag und die entscheidend damit zu tun hatte, daß die Insel im fernen Osten, anders als das Land in der Mitte Europas, ungeteilt aus dem Zweiten Weltkrieg herauskam. Die wiedervereinigungspolitischen Rücksichtnahmen auf Lasten der Vergangenheit, die für die Bundesrepublik daraus immer wieder erwuchsen, am offensichtlichsten vielleicht während der Zweiten Berlinkrise infolge der Hakenkreuzschmierereien an der Kölner Synagoge 1959, standen in einem denkbar krassen Gegensatz zur nationalpolitischen Position Japans.

Nicht nur daß Japan selbst nach 1945 ungeteilt und staatlich intakt geblieben war - obendrein erfuhren ausgerechnet zwei der Hauptopfer der ultranationalistischen Aggression, China und Korea, verbunden mit weiteren kriegerischen Auseinandersetzungen 1949 bzw. 1945/48 das Schicksal staat-

36 Fuchs, The American Kaleidoscope, S. 230 f.

37 Vgl. hierzu die Anmerkungen im Kapitel XI.

38 Vgl. Chang, Die Vergewaltigung von Nanking, S. 13.

39 Auch wenn der ökonomische und soziale Aufstieg der chinesischen Bevölkerungsgruppe in den USA seit dem Wendepunkt 1943 rasch Fortschritte machte. Vgl. Lyman, Chinese Americans. 
licher Trennung und damit auch außenpolitischer Schwächung. Zu den Hauptopfern der nationalsozialistischen Diktatur zählende Länder wie die Sowjetunion und Polen kannten ähnliche Probleme nicht. Sie bildeten national selbstbewußte Staaten, denen von Hitler-Deutschland die schwersten, noch lange brennenden Wunden in ihrer Geschichte zugefügt worden waren. Zwar hatten sie aus verschiedenen Gründen oft selbst keinerlei Interesse daran, eigenen Opfergruppen wie Juden, Kriegsgefangenen oder Zwangsarbeitern einen besonderen Platz in der nationalen Erinnerung einzuräumen oder ihnen gar im Verhältnis zur Bundesrepublik als Nachfolgestaat des Deutschen Reiches größeres Gewicht beizumessen ${ }^{40}$. Aber immerhin wurden im Rahmen eines Auslieferungsprogramms bis 1950 vor allem aus den Westzonen über 1800 NS-Täter, darunter Rudolf Höß und Erich Koch, nach Polen gebracht, um sie der Bestrafung zuzuführen ${ }^{41}$. Auch später waren die Staaten des Ostblocks, vor allem, aber nicht nur die DDR, schon aus Gründen kommunistischer Ideologie und antifaschistischer Systemlegitimation nach Kräften bemüht, die wirtschaftlich gefährlich erfolgreiche Bundesrepublik bei passender Gelegenheit auch und gerade mit öffentlichen Vorwürfen wegen einer „unbewältigten Vergangenheit“ politisch so weit wie möglich zu destabilisieren. Das reichte von der Warnung der Vertreter von 15 kommunistischen Parteien auf einem Ostberliner Treffen im Sommer 1958 gegen Atomwaffen in Händen „von Hitlergenerälen und SS-Offizieren“42 bis hin zu abenteuerlichen Kampagnen der „Iswestija“ etwa gegen Bundeskanzler Adenauer, der angeblich im Gästebuch der Washingtoner Nationalgalerie neben seiner Unterschrift Hakenkreuzzeichen angebracht haben sollte ${ }^{43}$. Solch wiederkehrende Attacken aus dem Osten verstärkten nur noch das Angewiesensein der Bundesrepublik auf die westlichen Partnerstaaten wie etwa während jener denkwürdigen UN-Vollversammlung 1960, als die Außenminister Griechenlands, Belgiens und der Niederlande die Bundesrepublik gegen Anwürfe des polnischen KP-Sekretärs und des tschechoslowakischen Außenministers in Schutz nahmen, wobei der Belgier ausdrücklich auf die Kronzeugenschaft seines von deutschen Truppen zweimal besetzten Landes verwies und sich überzeugt zeigte, daß ein neues Deutschland entstanden $\operatorname{sei}^{44}$.

$\mathrm{Zu}$ den unterschiedlichen innen- und außenpolitischen Rahmenbedingungen der "Vergangenheitsbewältigung“ in Deutschland und Japan zählt schließlich ein weiterer wichtiger Punkt, auch wenn er weniger im Bereich der harten Fakten angesiedelt, sondern im engeren Sinne erinnerungskultu-

$40 \mathrm{Vgl}$. etwa Bonwetsch, Sowjetische Zwangsarbeiter vor und nach 1945.

41 Musial, NS-Kriegsverbrecher vor polnischen Gerichten, S. $39 \mathrm{f}$.

42 Brochhagen, Nach Nürnberg, S. 228.

43 Vgl. hierzu die Replik im Bulletin der Bundesregierung v. 5. April 1961, S. 621, und v. 6. April, S. 635.

44 Bulletin der Bundesregierung, 8. Oktober 1960, S. 1835. 
reller Art ist: Vom Zweiten Weltkrieg werden - mit dem niederländischen Richter beim Tokioter Militärtribunal, Bernhard V. A. Röling, zu reden vor allem zwei Dinge erinnert: „Die deutschen Gaskammern und die amerikanischen Atombomben"45. Industrialisierter Massenmord an den Juden und der militärische Einsatz von Kernwaffen mit einer bis dahin unvorstellbaren Vernichtungskraft waren die eigentlich neuen Schreckensphänomene des Zweiten Weltkriegs ${ }^{46}$. Im einen Fall fungierten überwiegend Deutsche als Täter, im anderen wurden zumeist Japaner zu Opfern. Gewiß waren auch Deutsche gerade am Ende des Krieges im Zuge der Vertreibung und des Bombenkrieges zu Opfern geworden; doch die schrecklichen Massenverbrechen an Deutschen schienen an Grausamkeit nicht zu übertreffen, was man in der Menschheitsgeschichte an Bestialitäten von dem assyrischen Schlächter Assurnassirpal II. über den Völkermord an den Armeniern bis zur Bombardierung Coventrys bereits leidvoll kannte. Ihr Neuigkeitswert war - journalistisch gesprochen - geringer als der von Hiroshima, das mit den wieder und wieder gezeigten Bildern des Atompilzes zu einem dauernden Memento gegen den Krieg wurde.

Tatsächlich bedeutete die Vernichtung von Zivilbevölkerung durch eine Atombombe von exorbitanter Sprengkraft und Hitzeentwicklung, deren radioaktive Strahlung noch dazu lebensgefährliche Spätfolgen zeitigte, eine neue Dimension der Massenvernichtung. Diese hat zumindest in ihrer apokalyptischen Anmutung mit der industriell betriebenen Vergasung in Auschwitz und andernorts manches gemeinsam, auch wenn dabei nicht vergessen werden darf, daß die politische bzw. militärische Motivation für beide Taten höchst unterschiedlich war; daß es in einem Fall also immerhin um die Beendigung eines Krieges, wenn auch mit höchst fragwürdigen Mitteln ging ${ }^{47}$, im anderen um den Albtraum einer Rassenideologie, die mit bis dahin ungekannter krimineller Energie ihre unschuldigen Opfer noch in den entlegensten Winkeln des Herrschaftsbereichs aufspürte und die ihrer massenmörderischen Obsession nötigenfalls sogar existentielle kriegsstrategische Interessen unterordnete. Dieses Unsägliche von „Auschwitz" rückte die Rolle der Deutschen als Täter ganz in den Vordergrund der kollektiven

45 Röling, zit. nach Minear, Victor's Justice, S. 101.

46 Für die öffentliche Wahrnehmung gilt dies im Grunde bis heute, auch wenn die Forschung in den letzten Jahren verstärkt darauf aufmerksam gemacht hat, daß industrialisiertes Töten zuerst an psychisch Kranken ,erprobt“ wurde und der Holocaust nicht mit Auschwitz gleichgesetzt werden kann, weil eben nicht nur Millionen Menschen auf industrielle Weise ermordet, sondern Millionen andere vor allem im Osten auch auf dem „konventionelleren“ Weg der Massenerschießung u.ä. getötet wurden.

47 In einem von Opfern der Atomwaffen 1957 angestrengten Prozeß machte die japanische Regierung übrigens selbst geltend, der Abwurf der Bomben sei von internationalem Recht gedeckt gewesen. Der US-Senat hat im Verlauf einer jüngeren amerikanischen Kontroverse 1994 bekräftigt: „The role of the Enola Gay during the World War II was momentous in helping to bring World War II to a merciful end, which resulted in saving lives of Americans and Japanese“. Igarashi, Bodies of Memory, S. 219, Zitat S. 42. 
Erinnerung der Welt - und die große Vertreibung oder das Inferno von Hamburg, Dresden und Swinemünde nach hinten. Die japanischen Kriegsverbrechen dagegen konnten lange weitgehend hinter dem Atompilz von Hiroshima verschwinden.

In diese Richtung deuten nicht zuletzt Aussagen amerikanischer, japanischer, aber auch israelischer Provenienz, die atomaren und nationalsozialistischen Holocaust auf ein- und derselben Ebene ansiedeln ${ }^{48}$. Hier ist zunächst an Dwight Macdonalds Artikel in Politics vom August $1945 \mathrm{zu}$ erinnern, wo es hieß, die grausame Aktion der Atombombenabwürfe stelle „,uns', die Verteidiger der Zivilisation, auf eine moralische Stufe mit ,ihnen', den Bestien von Maidanek. "49 Der bekannte japanische Historiker Ienaga sprach 1968 von Auschwitz und Hiroshima als „klassischem Beispiel rationaler Greueltaten "50. Auch der Auschwitz-Überlebende Primo Levi hat in einem seiner Gedichte („Das Mädchen von Pompei“) einem Opfer des Vesuv-Ausbruchs und mit ihm Anne Frank sowie einer Schülerin von Hiroshima ein literarisches Denkmal gesetzt, d.h. vulkanischen, nationalsozialistischen und atomaren Holocaust in einen Zusammenhang gebracht. Im Mai 1994 schließlich sprach der israelische Außenminister Shimon Peres ohne großen Widerspruch zu ernten - bei einer Feier im UN-Hauptquartier in New York von „two holocausts: the Jewish holocaust and the Japanese holocaust. Because nuclear bombs are like flying holocausts. "51 Kein Zweifel also, so abwegig die Parallelisierung von Auschwitz und Hiroshima inhaltlich auch war, so viele Spuren hat sie in der globalen Erinnerungskultur doch hinterlassen. Und diese Folgen gehören nicht zuletzt zu dem komplexen Spannungsfeld, in dem sich die nationale „Vergangenheitsbewältigung“ in Japan und Deutschland vollzog.

48 Vgl. Minear, Atomic Holocaust, Nazi Holocaust, v.a. S. $354 \mathrm{ff}$.

49 Politics, August 1945, S. 225.

50 Ienaga, The Pacific War, S. $187 \mathrm{f}$.

51 Minear, Atomic Holocaust, Nazi holocaust, S. 356. Die Friedensglocke, an der Peres seine Rede gehalten hatte, war 1954 mit Steinen aus Israel und Holz aus Japan gebaut worden. 